\title{
SYNERGY OF ARTIFICIAL NEURAL NETWORKS AND KNOWLEDGE-BASED EXPERT SYSTEMS FOR INTELLIGENT FMS SCHEDULING
}

\author{
Luis Carlos Rabelo * \\ Sema Alptekin * \\ Ali S. Kiran **
}

*CIM Laboratory

Eng. Management Department

University of Missouri-Rolla

Rolla, Missouri 65401
**Industrial Eng. Department Univ. of Southern California Los Angeles, California

\section{ABSTRACT}

In this paper we describe a hybrid architecture that integrates artificial neural networks and knowledge-based expert systems to generate solutions for the real time scheduling of flexible manufacturing systems. The artificial neural networks perform pattern recognition and, due to their inherent characteristics, support the implementation of automated knowledge acquisition and refinement schemes through a feedback mechanism. The artificial neural network structures enable the system to recognize patterns in the tasks to be solved in order to select the best scheduling rule according to different demands. The knowledge-based expert systems are the higher order elements which drive the inference strategy and interpret the constraints and restrictions imposed by the upper levels of the flexible manufacturing system control hierarchy. The level of self-organization achieved provides a system with a higher probability of success than traditional approaches.

\section{INTRODUCTION}

Artificial neural networks (ANNs) are information/processing systems motivated by the goals of reproducing the cognitive processes and organizational models of neurobiological systems. As a result of their computational structure, ANNs feature attractive characteristics and have been applied successfully to a variety of problems. On the other hand, knowledge-based expert systems (KBES) technology has been concentrating on the construction of high performance programs in specialized and limited domains. Expert systems provide in their computational order explanation and justification capabilities, an efficient problem solving scheme, and a consistent validation strategy. KBES and ANNs might be integrated to solve tasks that require different problem solving modalities, such as feature extraction, data interpretation, distributed decision-making, optimization, constrained search. An example of a task whose concept welds several artificial intelligence (AI) disciplines is flexible manufacturing system (FMS) scheduling.

Flexible manufacturing systems (FMSs) are automated manufacturing systems consisting of numerical control (NC) 
machine tools, material handling devices, automated inspection stations, in-process storage areas, and a computational (hardware-software/processing-communications) scheme to provide databases handling, supervisory and monitoring functions. Flexible manufacturing systems are characterized by high flexibility and complexity.

Consequently, the scheduling of jobs, machines and other resources in FMS to achieve the production goals assigned, taking into consideration their decision making time frame, is a difficult task $[2,4]$.

In this paper, we present the design and implementation of an intelligent scheduling system for FMS (ISS/FMS) that utilizes AI technologies so that expected performance levels can be accomplished. ANNs are used as a method for predicting the behavior of the dispatching rules available in ISS/FMS rather than utilizing rules based on statistical models [11]. Their ability to learn from examples provides a self-acting strategy to the knowledge acquisition process and therefore a direct contribution to support self-organization schemes. KBES are utilized to interpret the goals and commands from the different elements of the hierarchical FMS architecture, interact with the user, monitor the performance and develop retraining strategies to enhance the artificial neural networks structures, and to implement sophisticated scheduling procedures. Distributed expert systems (DES) and distributed knowledge bases, especially in the case of the utilization of KBES to implement sophisticated scheduling procedures that are system specific, support a less constrained decision making environment allowing the intervention of these modules when they are essentials or the decision making time frame is long enough to permit their utilization.

\section{INTEGRATION OF ANNS AND KBES}

ANNs and KBES might be integrated in order to strengthen the best features of each. The pursuit of symbiotic system architectures which integrate multiple modalities may provide enhanced inferencing functionality and dynamic control of those architecture portions that change through time. Examples of some possible ways to integrate ANNs and KBES are explained below:

a. Development of innovative knowledge acquisition strategies using ANNs to extract and synthesize the knowledge. The knowledge acquired could be translated to rules and therefore the KBES would provide a better human-machine symbiotic system--justification and explanation mechanism support [8] .

b. KBES could be utilized to generate a first cut solution. The ANNs would optimize or utilize its learning and generalization properties to refine the strategy and perform the task with a higher efficiency [3].

c. KBES could be utilized to monitor the performance of ANNs and automate the learning of the ANN units. The KBES will generate procedures to modify and update the training files, and retrain the ANNs $[3,7,10]$.

d. ANNs would perform the tasks for which the KBES have performance degradations such as pattern recognition, hypertext retrieval, etc. $[2,7]$. 
e. Development of symbiotic methods which combine symbolic and neural techniques to enhance learning and inference mechanisms. The artificial neural modality will enhance the knowledge representation and the manipulation of uncertainty. On the other hand, the symbolic part will be used to handle the complexity dimension of the inference strategy [5].

\section{INTELLIGENT SCHEDULING SYSTEM FOR FMS (ISS/FMS) ARCHITECTURE}

ISS/FMS consists of the following units (See Figure 1) [7]: Expert Scheduler, Heuristics Programs, Artificial Neural System for Heuristics(ANSH), Expert Look-Ahead Scheduler, Artificial Neural System for Coefficients(ANSC), Learning Unit, Decision Support Unit, and databases.

This intelligent system has been designed using modularity principles and conveys ideas of distributed knowledge bases as do distributed expert systems. The architecture of the system is able to map computational structures implicating multitasking and moderately coupled parallelism.

\subsection{The Expert Scheduler}

The Expert Scheduler is the knowledge controller of ISS/FMS. In order to perform its high order functions the Expert scheduler consists of several rule-based modules (implemented in PROLOG) utilizing backward chaining and exercising backtracking, recursively generated sequences, and operating resources requests when it is deemed necessary. It is composed of the following rule-based modules: Interpretation and Feasibility, Controller, and Discriminator.

\subsubsection{Interpretation and Feasibility}

This rule-based module interprets the request from the upper hierarchical level for scheduling. This request is stated by commands and databases. The job database (See Table 1) and objectives to be achieved in this scheduling are analyzed using updated information on the current status, and the availability of resources and materials of the given FMS cell. If the information is incomplete, more complete information is requested from that specific level.

This rule-based module based on the job database, requested data, and degree of feasibility, decides to call the Artificial Neural System for Heuristics (ANSH) that will make a prediction of the behavior of each heuristic available to solve the problem. The current implementation of ISS/FMS is strictly based on tardiness.

\subsubsection{Controller}

The controller is a rule-based module in the Expert Scheduler that takes into consideration the output of the ANN(s). The controller is based on the request of the higher level and the relative heuristic ranking provided by the ANN(s). This criterion is used to call specific heuristic(s). Also, based on the time frame provided, the Expert Look Ahead Scheduler (ELAS) will be called. 


\subsubsection{Discriminator}

The Discriminator is a rule-based module in the Expert scheduler. The discriminator receives the answers to the scheduling problem from the selected heuristics and the Expert Look Ahead Scheduler (ELAS). It selects the best among them, and proceeds to send the answers to the appropriate levels. If the final schedule does not meet some of the high priority constraints, the Discriminator checks the decision time frame available to determine the time constraint. It then makes changes to the job database and recursively proceeds with the process.

\subsection{Heuristics Programs}

The dispatching rules utilized in this research prototype are:

SPT: Shortest Process Time;

EDD: Earliest Due Date;

CR: Critical Ratio;

SLACK: Slack Time Remaining;

S/OPN: Slack/Operation;

LWR: Least Work Remaining.

\subsection{Artificial Neural system for Heuristics (ANSH)}

The Artificial Neural System for Heuristics according to the parameters passed by the Interpretation and Feasibility module decides what ANN(s) to use. The Artificial Neural System for Heuristics has several ANNs based on the number of machines, number of jobs utilized, and performance measure desired. These are three-layer feedforward networks trained using the Backpropagation model [9].

\subsubsection{Input Feature space for Tardiness}

one has to develop appropriate ways to represent the problem to be learned in order to make it understandable for the network. Without this key feature, the neural network will fail to learn the appropriate relationship with the efficiency and accuracy desired. For the FMS scheduling and tardiness problem, the following dimensions were selected for the input feature space (See Figure 2):

1. Group Technology.

2. Time Remaining Until Due Date.

3. Number of Jobs.

In the current ISS/FMS research prototype data from an FMS cell with capacity to manufacture starting with three process plans has been selected.

\subsubsection{Training}

The ANNs were trained with data generated from simulations performed for that purpose using the Backpropagation model. A normal training session was able to spend in average more than 125 hours and using training data files of $92 \mathrm{~Kb}$ in IBM 6152 workstations. The ANNs have 15 input units, 65 hidden units (average), and 6 output units. 


\subsection{Expert Look Ahead scheduler (ELAS)}

This intelligent system implements a new feedback based heuristic scheduling procedure to generate optimal schedules. This heuristic procedure which was utilized mainly for the tardiness criterion $[4,6]$, has been proven to be efficient for the FMS scheduling problem. ELAS calls the Artificial Neural System for Coefficients (ANSC) which is utilized to provide coefficients to accelerate the performance of the algorithm and improve its efficiency. ELAS also calls the feedback heuristic algorithm and has a set of rules to make an intelligent search from the results provided by the algorithm using the coefficients predicted by ANSC. This rule-based module is only utilized if the decision time frame permits.

\subsubsection{Artificial Neural system For Coefficients (ANSC)}

The relationship between the job characteristics and the coefficients is very complex [8]. Also, the combinatorial explosion of the different job databases and the number of coefficients makes it prohibitive to utilize rules or other search techniques. It was found in preliminary simulations performed that 15 pairs of coefficients usually provide good findings (sufficient to make the algorithm achieve substantially better results than conventional heuristics). Extensive evaluation resulted in selection of 120 pairs. These 120 pairs increased the performance of the algorithm (See Table 2). The next step would be to provide a methodology with which to predict the behavior of the coefficients with certain levels of accuracy to select a pair or set of coefficients.

After the analysis described above, a neural network system for coefficients using the Backpropagation model was implemented. One of the ANNs tested was able to map the relationship with only 2,000 iterations. Scheduling problems out of the training set were shown to the network, and it was able to predict an optimum pair of coefficients in 90 percent of the cases. After evaluation of the results, several modifications were made to the network topologies to improve the performance.

As was explained with ANSH, several ANNs were trained using the 120 pairs mentioned above for different problem types rather than using a large ANN. These are three layer feedforward ANNs. Their average size is 15 input units, 50 hidden units and 22 ( 10 $* 12=120$ pairs) output units. ANSC operates in a manner similar to ANSH, using the same input feature space and for the same FMS cell attributes.

\subsection{Units Under Development}

The Decision Support Aid Unit and the Learning Unit in the current ISS/FMS research prototype are under development. They will provide innovative features to ISS/FMS.

The learning unit is another example of the integration of KBES and ANNs. This unit implements a feedback mechanism needed to identify how well the knowledge encoded in the different ANNs is performing. A KBES monitors the output of ANSC and ANSH. If discrepancies are found, the monitor KBES will identify the responsible unit and the new "data sample" will be added to the 
historical set, and a backup copy of that specific ANN will be retrained. This retraining can cause changes in the ANN architecture. Provisions to make modifications to this topology will be encoded in the monitor KBES which interacts closely with implementation of the Backpropagation paradigm, which is written in the $C$ programming language [7]. This part of the Learning Unit is in the implementation stage.

\section{AN EXAMPLE}

The following is an example that embodies some of the ideas behind ISS/FMS.

\subsection{Problem Definition}

Suppose that there is a type of three machine and eight-job scheduling problem. An FMS cell which has three basic products, and several specific delivery times per period is given.

\subsection{Training and Results}

First simulations were generated to develop a first data set of 400 data samples and an ANN was trained. An initial architecture was selected based on experience. When the initial architecture was unable to reach the desired performance, a new topology was selected.

Finally a successful topology was found. An additional 400 data samples were generated and the topology was trained with the 800 data samples. When the mapping was done, 800 data samples were generated and taught. When the formerly successful topology was unable to learn, it was replaced with a new one. Finally, a system with 15 input units, 70 hidden units, and 6 output units was able to learn the 1600 data samples. This network was given a new set of 200 job databases, was tested and was limited to only one answer (it was only allowed to select one rule). Its performance against conventional rules is shown in Table 3 . It was found that it chose the best rule in $92 \%$ of the cases and, in the remainder it was able to predict the second best (See Table 3).

\section{SUMMARY AND FUTURE ENHANCEMENTS}

ISS/FMS has been designed based on the integration of several technologies. Artificial neural networks has been utilized as an effective predicting tool and scheduling pattern recognition mechanism. on the other hand, knowledge-based expert systems have been utilized as the higher order members that interact with other elements of the FMS hierarchy providing guidance for problem solving strategy, monitoring the performance of the system, and automating the ANN learning process. As a result the level of responsiveness achieved may provide the necessary strength for scheduling in FMS. 


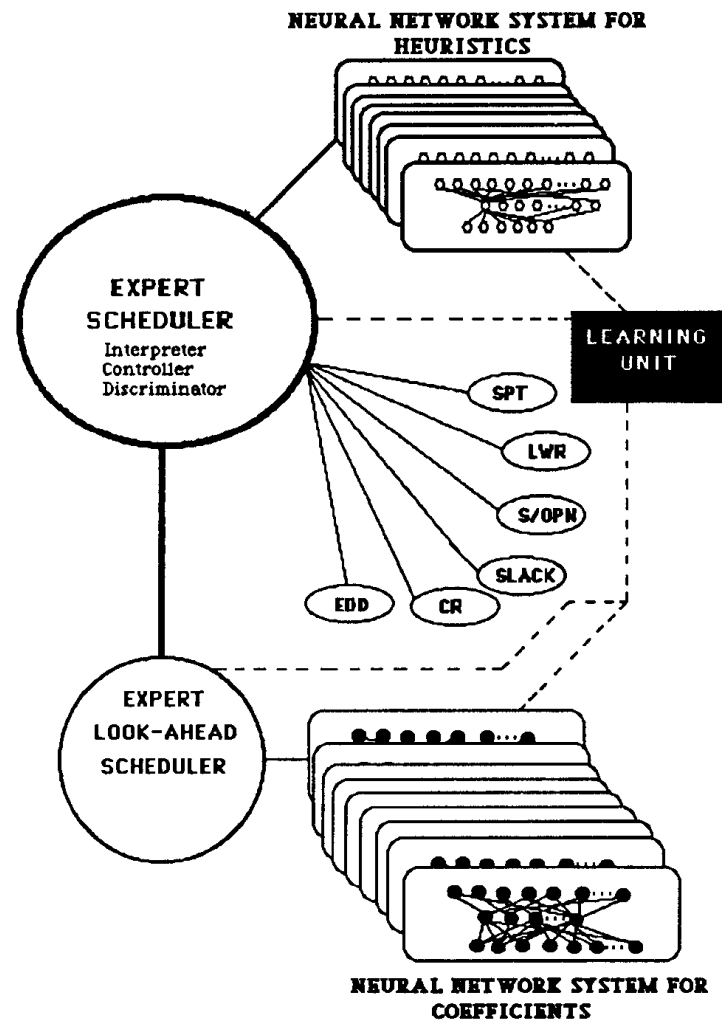

FIGURE 1. ISS/FMS ARCHITECTURE.

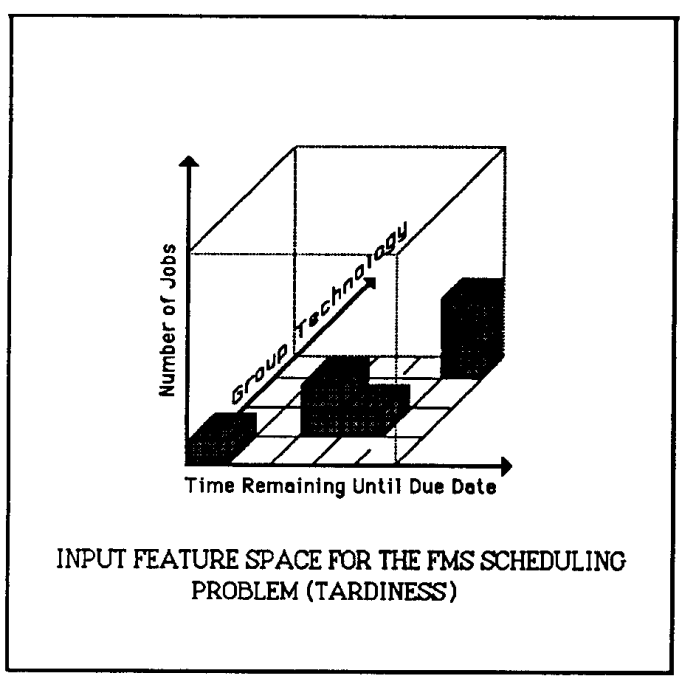

FIGURE 2. INPUT FEATURE SPACE.

\begin{tabular}{|c|c|c|c|c|c|}
\hline $\begin{array}{l}\text { JOB } \\
\text { NUMBER }\end{array}$ & $\begin{array}{l}\text { OPERATTON } \\
\text { NUMBER }\end{array}$ & $\begin{array}{l}\text { READY } \\
\text { TME }\end{array}$ & $\begin{array}{l}\text { DUE } \\
\text { DATE }\end{array}$ & $\begin{array}{l}\text { MACHINR } \\
\text { NUMBER }\end{array}$ & $\begin{array}{l}\text { UNIT } \\
\text { IIME }\end{array}$ \\
\hline 1 & $\begin{array}{l}1 \\
2 \\
3\end{array}$ & $\begin{array}{l}0 \\
0 \\
0\end{array}$ & $\begin{array}{l}26 \\
26 \\
26\end{array}$ & $\begin{array}{l}2 \\
2 \\
1\end{array}$ & $\begin{array}{l}4 \\
4 \\
8\end{array}$ \\
\hline 2 & $\begin{array}{l}1 \\
2\end{array}$ & $\begin{array}{l}0 \\
0\end{array}$ & $\begin{array}{l}23 \\
23\end{array}$ & $\begin{array}{l}3 \\
1\end{array}$ & $\begin{array}{l}7 \\
7\end{array}$ \\
\hline 3 & 1 & 0 & 19 & 3 & 6 \\
\hline 4 & $\begin{array}{l}1 \\
2\end{array}$ & $\begin{array}{l}0 \\
0\end{array}$ & $\begin{array}{l}14 \\
14\end{array}$ & $\begin{array}{l}1 \\
3\end{array}$ & $\begin{array}{l}3 \\
4\end{array}$ \\
\hline 5 & $\begin{array}{l}1 \\
2 \\
3\end{array}$ & $\begin{array}{l}0 \\
0 \\
0\end{array}$ & $\begin{array}{l}23 \\
23 \\
23\end{array}$ & $\begin{array}{l}2 \\
3 \\
2\end{array}$ & $\begin{array}{l}4 \\
3 \\
9\end{array}$ \\
\hline 6 & 1 & 0 & 11 & 3 & 5 \\
\hline 7 & $\begin{array}{l}1 \\
2\end{array}$ & $\begin{array}{l}0 \\
0\end{array}$ & $\begin{array}{l}26 \\
26\end{array}$ & $\begin{array}{l}2 \\
1\end{array}$ & $\mathbf{5}$ \\
\hline 8 & $\begin{array}{l}1 \\
2 \\
3\end{array}$ & $\begin{array}{l}0 \\
0 \\
0\end{array}$ & $\begin{array}{l}27 \\
27 \\
27\end{array}$ & $\begin{array}{l}1 \\
2 \\
3\end{array}$ & $\begin{array}{l}4 \\
5 \\
4\end{array}$ \\
\hline
\end{tabular}

TABLE 1. JOB DATABASE EXAMPLE.

FEEDBACK HEURISTIC (300 PROBLEMS) AVERAGE TARDINESS

\begin{tabular}{|cccc|}
\hline MACHINE & JOB & HF & HF \\
\hline 3 & 6 & 25.47 & 25.27 \\
3 & 8 & 30.78 & 30.35 \\
3 & 10 & 35.26 & 34.56 \\
\hline
\end{tabular}

FEEDBACK HEURISTIC ( 300 PROBLEMS) MINIMUM TARDINESS FREQUENCY

\begin{tabular}{|ll|}
\hline $\mathrm{HF}^{*}$ & $\mathrm{HF}^{*}$ \\
\hline 230 & 300 \\
\hline
\end{tabular}

HF * FEEDBACK heURISTIC ( 15 SELECTED PAIRS)

HF ${ }^{* *}$ FEEDBACK HEURISTIC (120 PAIRS)

TABLE 2. 15-PAIR VS 120-PAIR

\begin{tabular}{|c|c|r|r|r|r|r|}
\hline \multicolumn{6}{|c|}{ TEST (200 -NEW- PROBLEMS) } \\
\hline \multicolumn{1}{|c|}{ MINIMUM TARDINESS FREQUENCY } \\
\hline SPT & LWR & STR & SPO & CR & EDD & ANN \\
56 & 56 & 16 & 47 & 48 & 161 & 184 \\
\hline
\end{tabular}

TABLE 3. ANN AS A PREDICTING TOOL. 


\section{REFERENCES}

1. Bigus, J., and Goolsbey, K., "Integrating Neural Networks and Knowledge-Based Systems in a commercial Environment", Proceedings of the International Joint Conference on Neural Networks, Washington, D.C., 1990, pp. II463-II466.

2. Gross, J., "Intelligent Feedback Control for Flexible Manufacturing Systems," Ph. D. Thesis, University of Illinois at Urbana-Champaign, 1987.

3. Handelman, D., Lane, S., and Gelfand, S., "Integration of Knowledge-Based system and Neural Network Techniques for Autonomous Learning Machines," Proceedings of IJCNN, Washington, D. C., June $18-22,1989$, Published by IEEE TAB Neural Network Committee, 1989, pp. I683 - I688.

4. Kiran, A. and Alptekin, S., "A Tardiness Heuristic For Scheduling Flexible Manufacturing systems," 15th Conference on Production Research and Technology: Advances in Manufacturing systems Integration and processes, University of California at Berkeley, Berkeley, California, January 9-13, 1989, pp. 559-564 .

5. Myllimaki, P., Tirri, H., Floreen, P., and orponen, P., "Compiling High-Level Specifications into Neural Networks," Proceedings of the International Joint Conference on Neural Networks, Washington, D.C., 1990, pp. II475-II478.

6. Rabelo, L., "Comparison of Heuristics for Scheduling," Technical Report, Computer Integrated Manufacturing Laboratory, UMR, November 23, 1988.

7. Rabelo, L. and Alptekin, S., "Synergy of Neural Networks and Expert Systems for FMS Scheduling," Proceedings of the Third ORSA/TIMS Conference on Flexible Manufacturing Systems: Operations Research Models and Applications, Cambridge, Massachusetts, Elsevier Science Publishers B. V. , 1989, pp. 361-366.

8. Romaniuk, S., and Hall, L., " FUZzNET: Towards a Fuzzy Connectionist Expert System Developement Tool," Proceedings of the International Joint Conference on Neural Networks, Washington, D.C., 1990, pp. II483-II486.

9. Rumelhart, D., McClelland, J. and the PDP Research Group, Parallel Distributed Processing: Explorations in the Microstructure of Cognition, vol. 1: Foundations. Cambridge, MA: MIT Press/Bradford Books, 1988.

10. Schreinemakers, J., and Touretzky, D., "Interfacing a Neural Network with a Rule-Based Reasoner for Diagnosing Mastitis," Proceedings of the International Joint Conference on Neural Networks, Washington, D.C., 1990, pp. II 483-II486.

11. Thesen, A. and Lei, L. "An Expert System for Scheduling Robots in a Flexible Electroplating System with Dynamically Changing Workloads", Proceedings of the Second ORSA/TIMS Conference on FMS, Ann Arbor, Michigan, 1986. 